\title{
REFINED SOIL MOISTURE ESTIMATION BY MEANS OF L-BAND POLARIMETRY
}

\author{
Thomas Jagdhuber ${ }^{1}$, Irena Hajnsek ${ }^{1,2}$, Konstantinos P. Papathanassiou ${ }^{1}$ \\ ${ }^{1}$ German Aerospace Center, Microwaves and Radar Institute, PO Box 1116, 82234 Wessling \\ ${ }^{2}$ ETH Zurich, Institute of Environmental Engineering, Schafmattstr. 6, 8093 Zurich \\ Email: thomas.jagdhuber@dlr.de, irena.hajnsek@dlr.de, kostas.papathanassiou@dlr.de
}

\begin{abstract}
A recently published hybrid decomposition and inversion approach is adapted and enlarged using a generalized scattering model of the vegetation volume for a refined and more flexible soil moisture estimation under a temporally and spatially varying agricultural vegetation cover. Fully polarimetric SAR data of DLR's E-SAR system at L-band are used as observation basis. The results for the AgriSAR campaign, carried out in 2006 within the Peene catchment, reveal a detailed inversion due to the pixel-based procedure and very high inversion rates (>95\%) obtaining a gapless inversion along the entire growth cycle in strongly varying agricultural areas. The validation with in situ measurements for a plurality of summer and winter crops states a root mean square error of $4.55 \mathrm{vol} . \%$, while a wide moisture range ( 2-30vol.\%) is covered by the refined soil moisture inversion under vegetation using solely polarimetric SAR techniques.
\end{abstract}

Index Terms - Polarimetric SAR, L-band, soil moisture estimation under vegetation, hybrid decomposition

\section{INTRODUCTION}

As soil moisture is an Essential Climate Variables (ECV), its estimation in agricultural regions using active radar remote sensing is a research topic since several decades [1]. Especially the availability of Synthetic Aperture Radar (SAR) sensors operating at longer wavelength (L-band) provide the synergy of a sufficient penetration through the agricultural vegetation cover and a distinct backscattering signal to obtain a convenient signal-to-noise ratio. In addition, SAR polarimetry allows the investigation of the physical properties of the complex agricultural scatterers by observing the changes in polarimetry, when the electromagnetic waves interact with the different agricultural scatterers.

In agriculture, mainly three scattering contributions can be found at L-band: From the vegetation, from the underlying soil as well as from the combination of vegetation and underlying soil [2]. In this way, the acquired long wavelength data can be exploited with fully polarimetric decomposition techniques to separate agricultural vegetation from ground scattering components in order to extract the moisture characteristics of the underlying soil [3]. However, recent investigations on hybrid decompositions revealed that the combination of a model-based decomposition, (removal of the full-rank vegetation influence) with an eigen-based decomposition (separation of the lower-rank ground components) significantly eases the physicallybased extraction of the different ground scattering contributions (surface, dihedral) [4].

\section{REFINED METHODOLOGY OF SOIL MOISTURE RETRIEVAL UNDER VEGETATION COVER}

Hybrid, polarimetric decompositions represent one kind of innovative unmixing technique to separate different scattering contributions from one resolution cell of a fully polarimetric SAR acquisition [3]. In [4] a hybrid decomposition is presented, which only considers a random volume of dipoles as vegetation volume together with a physically constrained volume intensity component $f_{V}$. In order to enlarge the flexibility to describe a variety of vegetation volumes, the volume removal step within the hybrid decomposition is generalized as follows [3]:

$$
\left[T_{G}\right]=\left\langle\left[T_{\text {Data }}\right]\right\rangle-f_{V}\left[T_{V}\right]=\left[\begin{array}{ccc}
T_{11} & T_{12} & 0 \\
T_{12}^{*} & T_{22} & 0 \\
0 & 0 & T_{33}
\end{array}\right]-f_{V}\left[\begin{array}{ccc}
V_{11} & V_{12} & 0 \\
V_{12}^{*} & V_{22} & 0 \\
0 & 0 & V_{33}
\end{array}\right] \text { (1) }
$$

$\left[T_{G}\right],\left[T_{\text {Data }}\right]$ and $\left[T_{V}\right]$ represent the coherency matrices of the ground components (surface, dihedral), the acquired SAR data and the modeled reflection-symmetric vegetation volume, respectively. In this generalized hybrid decomposition the volume parameters $V_{11}-V_{33}$ can synthesize any kind of reflection symmetric volume scattering component. The most general case and the specific random volume case are defined in the following list [3]:

\begin{tabular}{ccc}
\hline Element & General case & $\begin{array}{c}\text { Random } \\
\text { volume case }\end{array}$ \\
\hline$V_{11}$ & $\frac{1}{2}\left(A_{p}+1\right)^{2} / 1+A_{p}^{2}$ & $\frac{1}{2}$ \\
$V_{12}$ & $\frac{1}{2}\left(A_{p}^{2}-1\right) \operatorname{sinc}(2 \Delta \psi) / 1+A_{p}^{2}$ & 0 \\
$V_{22}$ & $\frac{1}{4}\left(A_{p}-1\right)^{2}(1+\operatorname{sinc}(4 \Delta \psi)) / 1+A_{p}^{2}$ & $\frac{1}{4}$ \\
$V_{33}$ & $\frac{1}{4}\left(A_{p}-1\right)^{2}(1-\operatorname{sinc}(4 \Delta \psi)) / 1+A_{p}^{2}$ & $\frac{1}{4}$ \\
\hline
\end{tabular}


In the general case the geometry/shape of the vegetation volume is represented by the particle anisotropy $A p$ (from dipoles, discs to spheres), while the degree of orientation within the vegetation volume is described by the orientation distribution width $\Delta \psi$ (from totally oriented to randomly oriented volumes). In order to find the appropriate representation $(A p, \Delta \psi)$ of the vegetation volume within the range of possible solutions in Eq. 1, the $\alpha_{1}$-criterion, introduced in $[3,4]$ is adapted and enlarged by an $\alpha_{\text {min }}{ }^{-}$ criterion [5]. Both criteria are applied jointly and pixel-wise to find the best fitting combination of vegetation shape and orientation for each resolution cell. After proper volume removal the matrix of the two ground components $\left[T_{G}\right]$ is decomposed by an eigen-analysis and the polarimetric scattering alpha angles $\left(\alpha_{S}, \alpha_{D}\right)$ of the surface and dihedral component are retrieved by Eq. 2 . The physically meaningful allocation of $\alpha_{S}$ and $\alpha_{D}$ is guaranteed by an orthogonality criterion, first published in [6]. Together with the intensities of the surface and dihedral component $\left(f_{S}, f_{D}\right)$, being the eigenvalues of $\left[T_{G}\right]$ in Eq. 3, the decomposed ground components $\left(\alpha_{S}, \alpha_{D}, f_{S}, f_{D}\right)$ can be inverted for soil moisture by the inversion procedures developed in [2,3].

\section{EXPERIMENTAL RESULTS}

The refined moisture retrieval algorithm for agricultural vegetation cover is tested with the data set of the AgriSAR campaign. The AgriSAR campaign was conducted over four months in 2006 including the entire vegetation growth period [7]. The test site is located in Northern Germany within the Peene catchment. Within this campaign fully polarimetric L-band data were acquired by DLR's airborne E-SAR system with high spatial resolution (slant range: $1.5 \mathrm{~m}$, azimuth: $0.5 \mathrm{~m}$ ). Concurrently to the data acquisitions, soil moisture and vegetation parameters were measured on selected test fields with varying crop and soil types. Fig. 1 shows the data of a continuously recording soil moisture station, conducted by the LMU Munich for the entire growth period, together with the maximum vegetation height. The vertical blue bars represent the dates of the ESAR acquisitions, which track the decreasing trend in soil moisture from a level of 25vol.\% in April (beginning of growing season), to $15 \mathrm{vol} . \%$ in June down to 8vol.\% in July (end of growing season).
Hence, the retrieval algorithm is analyzed for a variety of soil conditions and for a distinctively growing vegetation cover along the growing season of 2006 (max. height: 18cm (April) - 172cm (July)), providing an optimum opportunity to study the soil moisture retrieval under agricultural vegetation cover.

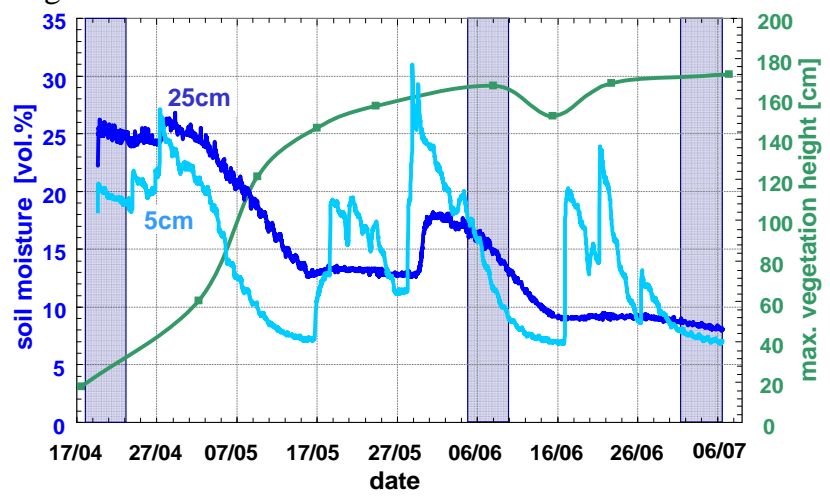

Fig. 1. Continuously recorded soil moisture in depth of $5 \mathrm{~cm}$ and $25 \mathrm{~cm}$ and maximum vegetation height for the entire growth period from April to July 2006, acquired by LMU during the AgriSAR campaign; Blue bars indicate data takes of the E-SAR system [7].

First results of the obtained orientation distribution $\Delta \psi$ from the novel generalized volume scattering model within the volume removal of the decomposition are shown exemplarily in Fig. 2 for the beginning (April), the middle (June) and the end (July) of the AgriSAR 2006 campaign.

As already described in detail in section 2, the refined hybrid decomposition (with a constraint volume intensity component $f_{v}$ ) allows a flexible adjustment to the varying vegetation volume characteristics, moving from more oriented in far range of the April scene (sparse/no canopy with flat, soil-covering plants) to almost entirely random oriented for the July scene (full canopy with high, voluminous plants).

The results for the soil moisture inversion under agricultural vegetation cover are displayed in Fig. 3 for the AgriSAR campaign (spatial averaging on $[T]$ : $10 \mathrm{~m} \times 10 \mathrm{~m}$ ). Despite the variety in hydrological and phenological conditions for the three acquisitions, which span over the entire growth season, the soil moisture inversion results in a gapless retrieval under a strongly varying agricultural vegetation cover (as seen in Fig. 1),

$$
\begin{aligned}
& \alpha_{S, D}=\arccos \left(\frac{1}{\sqrt{1+4\left|\frac{T_{12}^{*}-f_{V} V_{12}}{T_{11}-T_{22}-f_{V} V_{11}+f_{V} V_{22} \pm \delta}\right|^{2}}}\right) \quad \text { including } \\
& \delta=\sqrt{T_{11}^{2}+\left(T_{22}+f_{V} V_{11}\right)^{2}+4\left(T_{12}-f_{V} V_{12}\right)\left(T_{12}^{*}-f_{V} V_{12}\right)-2 T_{11}\left(T_{22}+f_{V}\left(V_{11}-V_{22}\right)\right)-2 f_{V}\left(T_{22}+f_{V} V_{11}\right) V_{22}+f_{V}^{2} V_{22}^{2}} \\
& f_{d, s}=\frac{1}{2}\left(T_{11}+T_{22}-f_{V} V_{11}-f_{V} V_{22} \pm \sqrt{\mid-4\left(T_{22}\left(T_{11}-f_{V} V_{11}\right)+\left(T_{12}-f_{V} V_{12}\right)\left(f_{V} V_{12}-T_{12}^{*}\right)+f_{V}\left(f_{V} V_{11}-T_{11}\right) V_{22}\right)+\left(T_{11}+T_{22}-f_{V}\left(V_{11}+V_{22}\right)\right)^{2}}\right)
\end{aligned}
$$



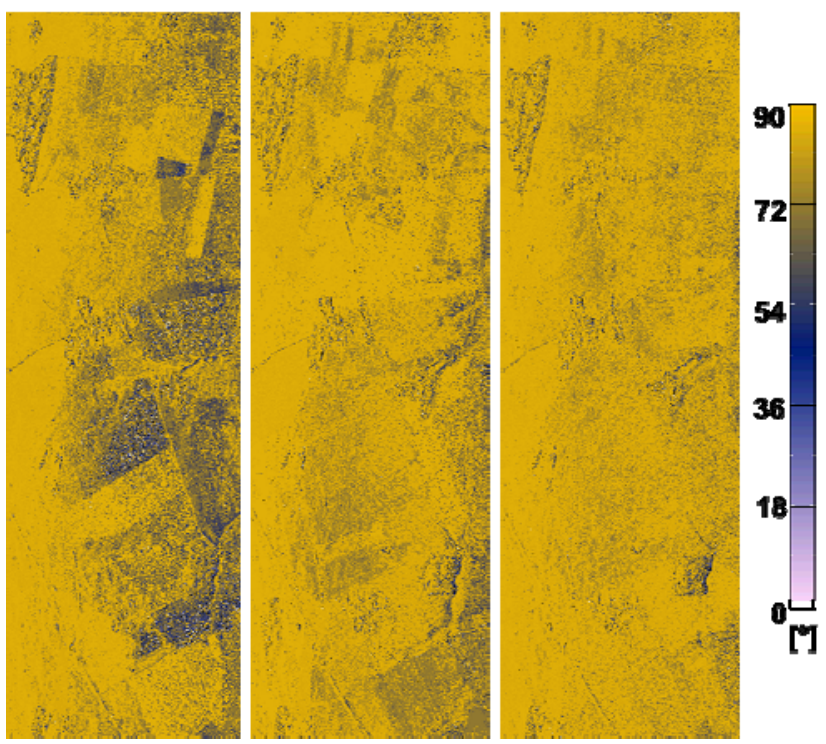

Fig. 2. Orientation distribution width $\Delta \psi$ (from totally oriented $\left(0^{\circ}\right)$ to randomly oriented volumes $\left.\left(90^{\circ}\right)\right)$ of the April (left), June (middle) and July (right) acquisition of the AgriSAR campaign.

while urban and forested areas are shaded gray due to scattering model invalidities. The achieved inversion rates are noted in Tab. 1 together with the estimated dielectric level required within the hybrid decomposition and inversion algorithm (see details in [3]) and with the maximum vegetation height, stating a distinct vegetation growth (from April to July) to a mature vegetation cover.

Tab. 1. $\varepsilon_{\text {est }}$-level, inversion rate and maximum of vegetation height for the different dates and acquisitions along the vegetation growth cycle

\begin{tabular}{cccc}
\hline Date & $\begin{array}{c}\varepsilon_{\text {est }} \text {-level } \\
{[-]}\end{array}$ & $\begin{array}{c}\text { Inversion } \\
\text { rate [\%] }\end{array}$ & $\begin{array}{c}\text { Max. vegetation } \\
\text { height [cm] }\end{array}$ \\
\hline $\begin{array}{c}\text { AgriSAR } \\
\text { April 2006 }\end{array}$ & 20 & 95.79 & 18 \\
$\begin{array}{c}\text { AgriSAR } \\
\text { June 2006 } \\
\text { AgriSAR }\end{array}$ & 10 & 99.98 & 167 \\
July 2006 & 8 & 99.99 & 172 \\
\hline
\end{tabular}

The quality of the soil moisture inversion under agricultural vegetation cover is assessed by a validation with in situ measurements of soil moisture in Fig. 4. For validation, FDR, TDR and gravimetric probes were used during the field campaigns to quantify the soil moisture content for representative measurement locations on several test fields with different types of winter crops (wheat, rape) and summer crops (sugar beet, corn).
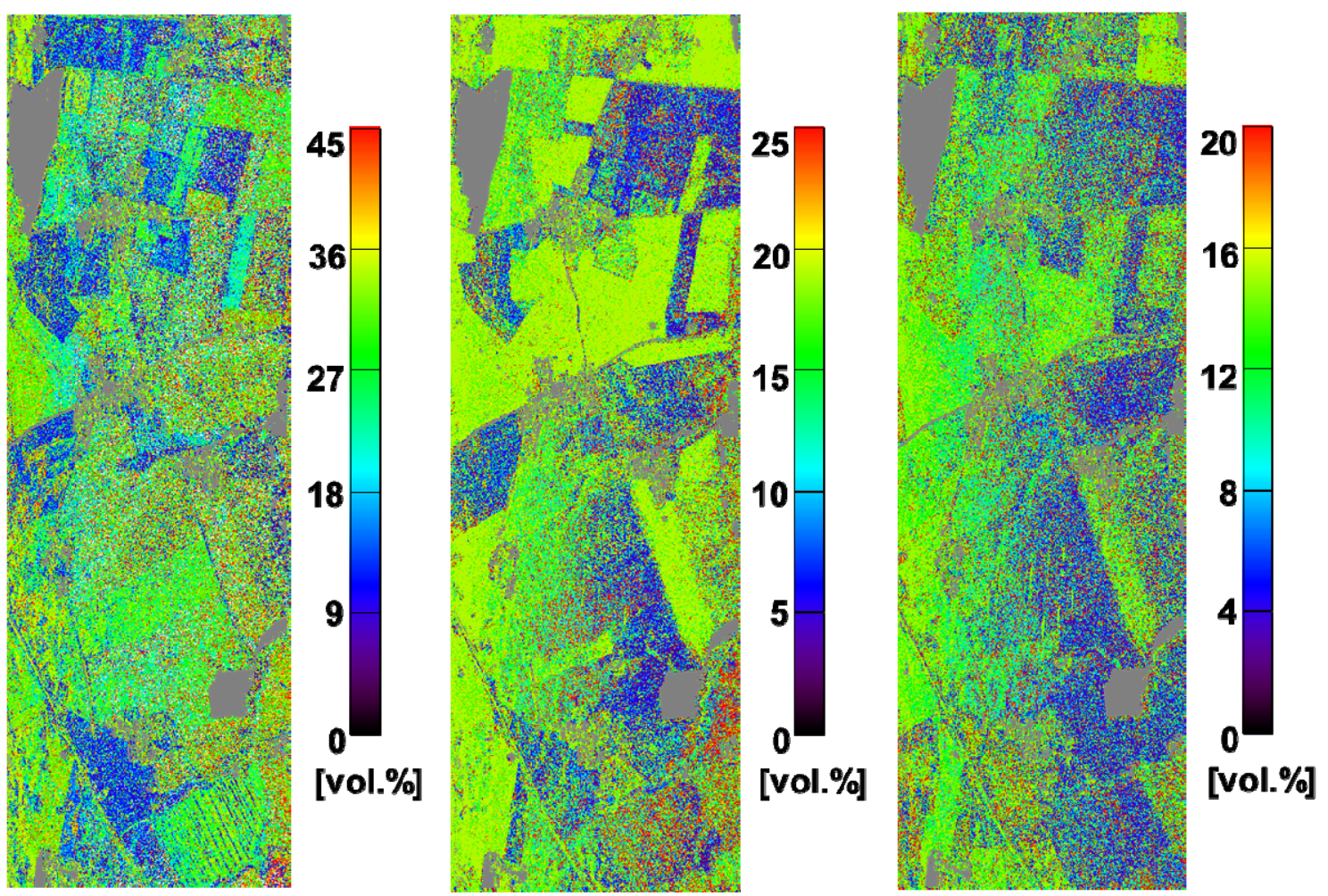

Fig. 3. Results of soil moisture inversion under agricultural vegetation in vol.\% using the refined hybrid decomposition and inversion approach for the three acquisitions covering the entire growing cycle (from left to right): AgriSAR in April, June and July 2006; White / gray color $=$ Non-invertible $/$ urban and forested areas. 
A box of 13x13 pixels was considered for each measurement location to realize 169 looks. Afterwards the mean value of each crop field is compared with the respective mean of the in situ measurements on the fields to yield the scatter plots for all (top of Fig. 4), winter (middle of Fig. 4) and summer (bottom of Fig. 4) crops.

The root mean square error for the variety of summer and winter crops amounts in total to $4.55 \mathrm{vol} . \%$ (winter: 4.09vol.\%; summer: 5.38vol.\%), while a wide range of measured soil moisture values from $\sim 2 \mathrm{vol} . \%$ to $~ 30 \mathrm{vol} . \%$ is covered for testing the inversion. With the novel volume generalization all crop type geometries (e.g. stalk-dominated or broad-leaf dominated) are incorporated well. Therefore no particular crop type deviates distinctively the soil moisture inversion performance.

\section{SUMMARY AND CONCLUSIONS}

Soil moisture estimates are obtained under agricultural vegetation for the entire growing season with a refined hybrid decomposition and inversion algorithm using fully polarimetric SAR data at L-band. The results for the AgriSAR campaign indicate very high inversion rates (>95\%) including all crop types. This leads to a spatially gapless inversion in agricultural areas. The validation with in situ measurements (TDR, FDR, gravimetric probes) for a variety of summer and winter crops results in a root mean square error of $4.55 \mathrm{vol} . \%$ (summer crops: $5.38 \mathrm{vol} . \%$; winter crops: $4.09 \mathrm{vol} . \%$ ). Almost all values in Fig. 4 are located close to the 1:1 line (solid line) indicating a high correlation independent of the type of vegetation cover with its different plant geometries and growing phenology.

\section{ACKNOWLEDGMENTS}

The authors would like to thank the AgriSAR, campaign members for their great cooperation, including especially the provision of the field data from the test site to validate the soil moisture retrieval algorithm. The in situ measurement systems were funded by ESA.

\section{REFERENCES}

[1] W. Wagner, G. Blöschl, P. Pampaloni, J.-C. Calvet, B. Bizzarri, J.-P. Wigneron \& Y. Kerr, "Operational readiness of microwave remote sensing of soil moisture for hydrologic applications,” Nordic Hydrology, vol. 38, pp. 1-20, 2007.

[2] I. Hajnsek, T. Jagdhuber, H. Schön \& K.P. Papathanassiou, "Potential of Estimating Soil Moisture under Vegetation Cover by means of PolSAR," IEEE Trans. Geosci. Remote Sensing, vol. 47, pp. 442-454, 2009.

[3] T. Jagdhuber, "Soil Parameter Retrieval under Vegetation Cover Using SAR Polarimetry,” $\mathrm{PhD}$ Thesis, University Potsdam, Potsdam, Germany, http://nbn-resolving.de/urn:nbn:de:kobv:517opus-60519, July 2012.

[4] T. Jagdhuber, I. Hajnsek, K.P. Papathanassiou, A. Bronstert, "Soil Moisture Retrieval Under Agricultural Vegetation Using Fully Polarimetric SAR," Proc. of IEEE International Geoscience and Remote Sensing Symposium, Munich, Germany, July 22-27, pp. 1481-1484, 2012.
[5] T. Jagdhuber, I. Hajnsek, K.P. Papathanassiou, "Polarimetric Decompositions for Soil Moisture Retrieval from Vegetated Soils in TERENO Observatories." Proc. of the $6^{\text {th }}$ PolInSAR conference, ESA-ESRIN, Frascati, Italy, January 28- February 1, 2013, p. 1-6. [6] S.R. Cloude, Polarisation: Applications in Remote Sensing," Oxford, Oxford University Press, 2010.

[7] R. Bianchi, M. Davidson, I. Hajnsek, M. Wooding \& C. Wloczyk, "AgriSAR 2006 - Final report," ESA final report, Noordwijk, The Netherlands, 2008.
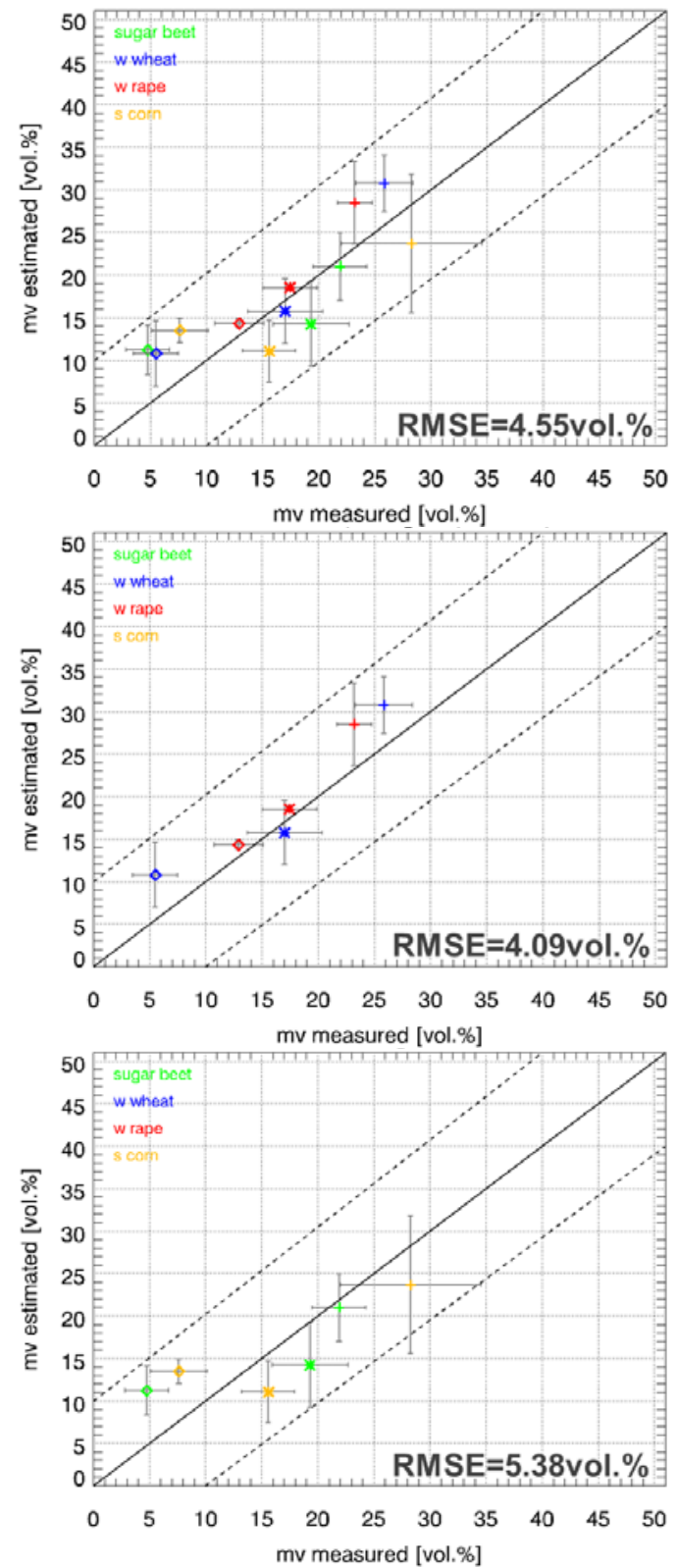

Fig. 4. Validation of estimated soil moisture values in April (+), June $(*)$ and July $(\diamond)$ for all crops (top), winter crops (bottom; blue: winter wheat, red: winter rape) and summer crops (middle; green: sugar beet, yellow: summer corn) with measured soil moisture values from FDR, TDR and gravimetric probes (symbols represent field mean values, gray bars indicate standard deviation on the field). 